\title{
PROCESSAMENTO, ESTABILIDADE E ACEITABILIDADE DE MARINADO DE VONGOLE (Anomalocardia brasiliana) ${ }^{1}$
}

\author{
Eliete da Silva BISPO ${ }^{2, *}$, Ligia Regina R. de SANTANA ${ }^{3}$, Rosemary D. S. CARVALHO ${ }^{2}$, \\ Clicia Capibaribe LEITE ${ }^{2}$, Maria Antonia Carvalho LIMA ${ }^{4}$
}

\begin{abstract}
RESUMO
O vongole (Anomalocardia brasiliana) tem importância socioeconômica para grande parte da população da região da Baía de Todos os Santos, na costa da Bahia, por fazer parte da dieta familiar e ser uma das principais fontes de renda. Para ampliar a comercialização deste molusco foi desenvolvido um processo de obtenção de marinado, envolvendo acidificação do produto com vinagre (pH $\leq 4,5)$ e tratamento térmico convencional (banho-maria) durante 30 minutos. O Índice de Aceitabilidade do marinado foi de 78-82\%, em relação à aparência, cor, aroma, sabor e textura. Sob os pontos-de-vista microbiológico, físico-químico, químico e sensorial, o marinado de vongole manteve-se estável durante 240 dias de armazenamento, a temperatura ambiente. O processo proposto atende a possibilidade de um maior e melhor aproveitamento comercial do vongole.

Palavras-chave: Anomalocardia brasiliana; vongole; marinado; processamento; estabilidade.
\end{abstract}

\section{SUMMARY}

PROCESSING, STABILITY AND ACCEPTABILITY OF MARINADE OF VONGOLE (Anomalocardia brasiliana). The vongole has economicsocial importance to the most of people in the "Todos os Santos" Bay, located in Bahia coast, Brazil; it is a product of the familiar diet and it is one of the principal rental source of this local people. In purpose to increase the mollusc sale was developed studies on the marinating of vongole, through acidification of the product with vinegar at a $\mathrm{pH}[4,5$ and a boiling water treatment during 30 min. The product was stored at room temperature for 240 days and the shelf life study included microbiological, physico-chemical, chemical and sensory evaluation. The process was effective to stablish commercial sterility to the marinaded vongole. According to sensory analyses, the marinaded vongole reached acceptability index like $78-82 \%$ for the overall appearance, colour, odour, flavour and texture. The data showed that the marinade of vongole was stable during storage for 240 days at room temperature.

Keywords: Anomalocardia brasiliana; vongole; marinade; processing; stability.

\section{1 - INTRODUÇÃO}

Grande parte da população da região da Baía de Todos os Santos, na costa da Bahia, sobrevive do extrativismo de mariscos - moluscos e crustáceos. Os mariscos têm importância nutricional. Caracterizamse como fontes expressivas de proteína e minerais, assim como são alimentos de baixo valor em calorias [12]. O vongole (Anomalocardia brasiliana), molusco capturado unicamente por mulheres, tem importância socioeconômica. Faz parte da dieta familiar e é uma das principais fontes de renda dessa população.

Essa exploração do vongole, todavia, não conta com procedimentos tecnológicos que sejam, como deveriam ser, simples, eficientes e capazes de conferir inocuidade ao produto. $\mathrm{O}$ molusco tem sido submetido a um rápido processo de cocção, retirado da concha e acondicionado em saco plástico, para depois ser transportado sob congelamento e comercializado em feiras-livres e supermercados.

1. Recebido para publicação em 09/08/2002. Aceito para publicação em 21/06/2004 (000952).

2. Faculdade de Farmácia - Universidade Federal da Bahia - UFBA, Rua Barão de Geremoabo, s/nº, Campus de Ondina, CEP: 40170-210, Salvador-BA.E-mail:ebispo@ufba.br; clicia@ufba.br

3. Departamento de Ciências da Vida - Curso de Nutrição - UNEB, Estrada das Barreiras, $s / n^{\circ}$, Narandiba/Cabula, CEP: 41195-001, SalvadorBA.E-mail: ligiarrs@bol.com.br

4. PIBIC - Faculdade de Engenharia de Alimentos - UEFS, CEP: 40000000, Feira de Santana-BA. E-mail: airamcarvalho@ufs.br

* A quem a correspondência deve ser enviada.
O processo de marinação do vongole pode possibilitar a segurança necessária ao consumidor e, ao mesmo tempo, garantir vida-útil adequada ao produto. Este processo prevê o uso de salmoura, geralmente composta de vinagre, vinho, azeite e especiarias [3].

O presente trabalho teve como objetivo estudar o processamento de marinado de vongole, avaliando a estabilidade e aceitabilidade do produto obtido.

\section{2 - MATERIAL E MÉTODOS}

\section{1 - Matéria-prima}

As amostras de vongole (Anomalocardia brasiliana) congeladas foram provenientes de Salinas das Margaridas-BA.

\section{2 - Avaliação microbiológica}

Os microrganismos coliformes totais, coliformes fecais, contagem total de aeróbios mesófilos, Bacillus cereus, bolores e leveduras, Staphylococcus aureus, Clostridios sulfito-redutores e Salmonella spp (em $25 \mathrm{~g}$ de amostra) foram avaliados segundo a AMERICAN PUBLIC HEALTH ASSOCIATION [1].

\section{3 - Avaliações físico-química e química}

$\mathrm{O} \mathrm{pH}$ foi determinado por leitura direta em potenciômetro e a acidez total titulável de acordo com a A.O.A.C.[2]. A rancidez (reação de Kreiss) e o índice de peróxido foram determinados conforme o recomendado pelo INSTITUTO ADOLFO LUTZ [9]. 


\section{4 - Processamento do marinado}

Foram realizados ensaios de formulação para o marinado de vongole, na tentativa de padronizar as quantidades de ácido (vinagre), azeite, sal ( $\mathrm{NaCl})$ e condimentos. Com o objetivo de tornar a tecnologia de obtenção do marinado facilmente reprodutivel por microempresas, foram utilizadas matérias-primas comuns. A acidificação do produto $(\mathrm{pH} \leq 4,5)$ foi feita com vinagre de vinho branco. Os demais condimentos eram sensorialmente compativeis com a matéria-prima. O processamento foi feito de acordo com a Figura 1, tendo por base a literatura [7].

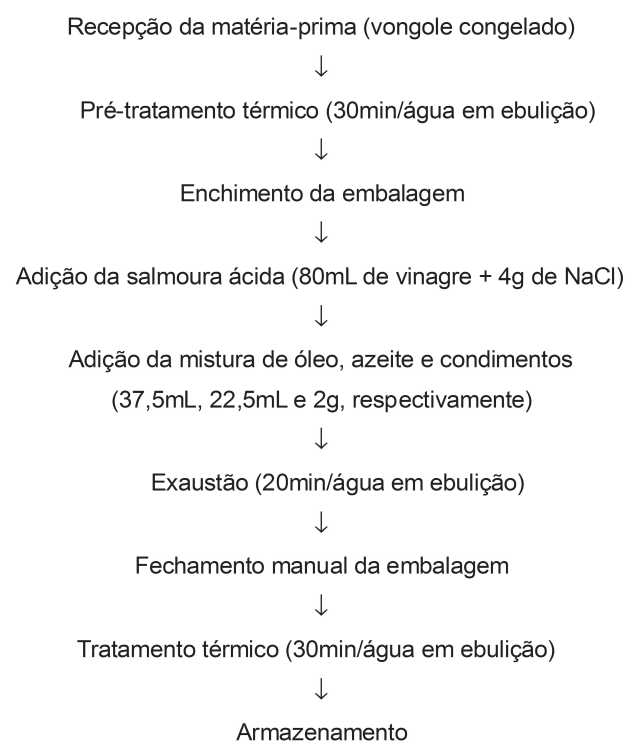

FIGURA 1. Processamento de marinado de vongole.

A matéria-prima foi aquecida em água à ebulição adicionada já nesta temperatura, e em volume suficiente para cobrir a superficie do produto, minimizando assim a perda de substâncias solúveis. Após a drenagem da água, o produto cozido foi acondicionado em frasco de vidro $(300 \mathrm{~mL})$, previamente esterilizado e adicionado de salmoura ácida, em temperatura de ebulição. Os demais componentes da formulação foram adicionados a temperatura ambiente, respeitando uma altura para o espaço livre no interior da embalagem.

Para a exaustão, o produto embalado, com a tampa semi-fechada, foi mantido parcialmente mergulhado em água à ebulição pelo tempo indicado, contado a partir do momento em que a salmoura atingiu $90^{\circ} \mathrm{C}$. O tratamento térmico final ocorreu com a embalagem completamente fechada, e o resfriamento subseqüente, com água inicialmente ao redor de $60^{\circ} \mathrm{C}$ e depois próxima de $38^{\circ} \mathrm{C}$. Finalmente, o produto obtido foi mantido a temperatura ambiente na faixa de $25-27^{\circ} \mathrm{C}$.

\section{5 - Aceitabilidade do marinado}

O Índice de Aceitabilidade (IA) foi realizado em relação aos atributos aparência global, cor, aroma, sabor e textura [13]. Trinta provadores avaliaram o quanto gostaram ou desgostaram do produto, utilizando escala hedônica estruturada de nove pontos, indo de 9 igual a "gostei extremamente" até 1 igual a "desgostei extremamente" [11]. A equipe de provadores, formada por homens e mulheres na faixa etária entre 18 a 50 anos, foi selecionada em função de consumirem produtos de mariscos, disponibilidade e interesse em participar do teste. O produto foi servido em recipientes brancos de polietileno, na porção de $10 \mathrm{~g}$ e a temperatura ambiente [10].

Para o cálculo do Índice de Aceitabilidade do produto foi adotada a expressão:

$\mathrm{IA}(\%)=\mathrm{A} \times 100 / \mathrm{B}$, onde $\mathrm{A}=$ nota média obtida para o produto, e B = nota máxima dada ao produto. O IA com boa repercussão tem sido considerado $\geq 70 \%[6,11]$.

\section{6 - Estabilidade do marinado}

Foi avaliada sob os pontos-de-vista físico-químico, químico, microbiológico e sensorial, durante 240 dias de armazenamento a temperatura ambiente, em intervalos de 30 dias.

Para a avaliação sensorial, foi treinada uma equipe de provadores, sendo feito, primeiramente, um levantamento da terminologia descritiva do produto, com base em MEILGAARD, CIVILLE \& CARR [10]. Após cada provador ter gerado seus termos descritivos para o produto, a equipe reuniu-se e, sob a supervisão de um lider, discutiu os termos levantados. De forma consensual, foi gerada uma lista de termos descritivos e com referências de intensidade definidas para os extremos da escala utilizada, conforme a Figura 2.

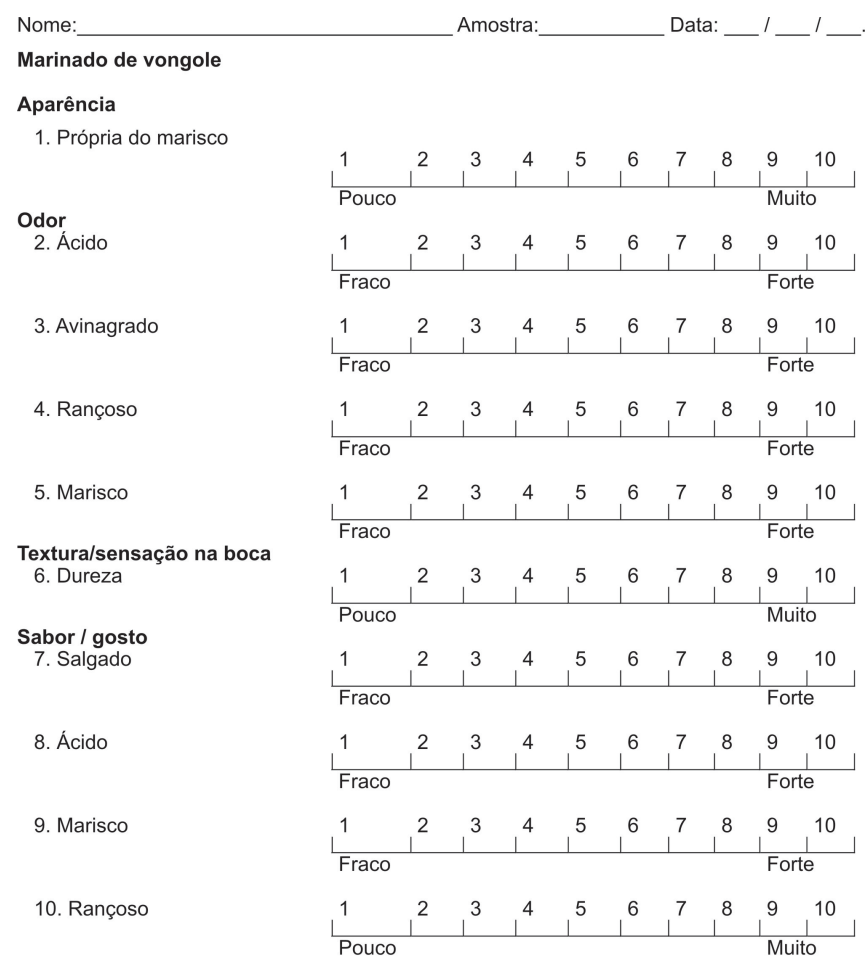

FIGURA 2. Ficha de avaliação sensorial do estudo de estabilidade do marinado de vongole. 


\section{7 - Análise estatística}

A análise estatística foi realizada empregando-se o delineamento de blocos completos casualizados. Os dados foram submetidos à análise de variância (ANOVA), para avaliar a existência de diferenças significativas. Estas diferenças foram analisadas através do teste de Tukey para comparação de médias, ao nivel de erro de $5 \%[8]$.

\section{3 - RESULTADOS E DISCUSSÃO}

\section{1 - Avaliação microbiológica, físico-química e quí- mica da matéria-prima e do marinado incubado}

Os resultados microbiológicos da matéria-prima e do marinado de vongole incubado estiveram de acordo com os padrões legais [4], indicando eficiência do processamento empregado (Tabela 1). O pH e a acidez do marinado confirmaram a adição de vinagre, enquanto o indice de peróxido não revelou processo oxidativo [5].

TABELA 1. Análises microbiológicas, físico-químicas e químicas da matéria-prima e do marinado de vongole incubado, durante 10 dias a $35^{\circ} \mathrm{C}$.

\begin{tabular}{lcc}
\hline Análises & Matéria-prima $\left(^{*}\right)$ & Marinado incubado $\left(^{*}\right)$ \\
\hline Aeróbios mesófilos totais(UFC/g) & $4,3 \times 10^{2}$ & ausente \\
Coliformes totais (NMP/g) ${ }^{*}$ & 3,0 & $<3,0$ \\
Coliformes fecais (NMP/g) ${ }^{\#}$ & 3,0 & $<3,0$ \\
Salmonella spp, em $25 \mathrm{~g}$ & ausente & ausente \\
Bacillus cereus (UFC/g) ${ }^{*}$ & ausente & ausente \\
Staphylococcus aureus (UFC/g) & ausente & ausente \\
Clostridios sulfito-redutores & ausente & ausente \\
Bolores e Leveduras (UFC/g) & ausente & ausente \\
pH & 6,4 & 4,43 \\
Acidez total titulável (\% ác. acético) & n.r. & 0,94 \\
Rancidez (Reação de Kreiss) & negativa & negativa \\
Índice de peróxido (mEq/Kg) & n.r. & 3,34 \\
\hline
\end{tabular}

(*) Resultados médios de cinco amostras $(\mathrm{n}=5)$.

(•) UFC/g - unidades formadoras de colônias por grama.

n.r. - não realizada.

\section{2 - Aceitabilidade do marinado}

Foi significativo o Índice de Aceitabilidade (IA) verificado para o marinado de vongole, entre 77,8 e $82,2 \%$, em cada atributo avaliado (Tabela 2), considerando a repercussão favorável quando $\geq 70 \%$, segundo a literatura $[6,11]$.

TABELA 2. Índice de Aceitabilidade do marinado de vongole, segundo cada atributo avaliado.

\begin{tabular}{lcc}
\hline Atributos & Notas Médias & Indice de Aceitabilidade (\%) \\
\hline Aparência & 7,0 & 77,8 \\
Cor & 7,0 & 77,8 \\
Aroma & 7,1 & 78,9 \\
Sabor & 7,2 & 80,0 \\
Textura & 7,4 & 82,2 \\
\hline
\end{tabular}

A cor natural do vongole sendo cinza-pardacenta é pouco atrativa. Eventualmente a correção da cor do produto, por meios tecnológicos, poderia proporcionar um IA mais elevado, com reflexos na aparência. Nesse sentido poderiam ser empregados condimentos disponiveis em cores apropriadas.

\section{3 - Estabilidade do marinado}

Os resultados das avaliações microbiológica, físico-química e química das amostras do marinado de vongole indicaram que o produto manteve-se estável a temperatura ambiente, durante 240 dias de armazenamento (Tabela 3).

TABELA 3. Análises microbiológicas, físico-químicas e químicas do marinado de vongole, durante 240 dias de armazenamento, a temperatura ambiente.

\begin{tabular}{|c|c|c|c|c|c|c|c|c|c|}
\hline \multirow{2}{*}{ Análises $\left(^{*}\right)$} & \multicolumn{9}{|c|}{ Armazenamento (dias) } \\
\hline & 01 & 30 & 60 & 90 & 120 & 150 & 180 & 210 & 240 \\
\hline \multicolumn{10}{|c|}{ Aeróbios mesófilos totais( $\mathrm{UFC} / \mathrm{g})^{\bullet}$ ausente ausente ausente ausente ausente ausente ausente ausente ausente } \\
\hline Coliformes totais $(\mathrm{NMP} / \mathrm{g})^{\#}$ & $<3,0$ & $<3,0$ & $<3,0$ & $<3,0$ & $<3,0$ & $<3,0$ & $<3,0$ & $<3,0$ & $<3,0$ \\
\hline Coliformes fecais $(\mathrm{NMP} / \mathrm{g})^{\#}$ & $<3,0$ & $<3,0$ & $<3,0$ & $<3,0$ & $<3,0$ & $<3,0$ & $<3,0$ & $<3,0$ & $<3,0$ \\
\hline Salmonella spp, em 25g & \multicolumn{9}{|c|}{ ausente ausente ausente ausente ausente ausente ausente ausente ausente } \\
\hline Bacillus cereus (UFC/g) ${ }^{*}$ & \multicolumn{9}{|c|}{ ausente ausente ausente ausente ausente ausente ausente ausente ausente } \\
\hline Staphylococcus aureus $(\mathrm{UFC} / \mathrm{g})^{*}$ & \multicolumn{9}{|c|}{ ausente ausente ausente ausente ausente ausente ausente ausente ausente } \\
\hline Clostridio sulfito-redutor & \multicolumn{9}{|c|}{ ausente ausente ausente ausente ausente ausente ausente ausente ausente } \\
\hline Bolores e Leveduras (UFC/g) ${ }^{*}$ & \multicolumn{9}{|c|}{ ausente ausente ausente ausente ausente ausente ausente ausente ausente } \\
\hline $\mathrm{pH}$ & 4,37 & 4,45 & 4,43 & 4,45 & 4,45 & 4,45 & 4,45 & 4,45 & 4,45 \\
\hline Acidez total titulável (\%ác. acético) & 1,18 & 1,13 & 1,14 & 1,11 & 1,12 & 1,06 & 1,05 & 1,08 & 1,08 \\
\hline Rancidez (Reação de Kreiss) & negat. & negat. & negat. & negat. & negat. & negat. & negat. & negat. & negat. \\
\hline Índice peróxido (mEq/Kg) & 3,29 & 3,30 & 3,30 & 4,28 & 4,95 & 4,90 & 4,90 & 4,98 & 4,98 \\
\hline
\end{tabular}

Os valores de $\mathrm{pH}$ e de acidez praticamente não sofreram modificação durante o experimento, garantindo a estabilidade microbiológica do produto. $\mathrm{O}$ índice de peróxido e a reação de Kreiss demonstraram que não houve alteração lipídica no produto (Tabela 3). Os valores encontrados de peróxidos estão de acordo com o padrão do Ministério da Saúde, que estabelece o limite máximo de $10 \mathrm{mEq} / \mathrm{kg}$ para óleos e gorduras refinadas e de $20 \mathrm{mEq} / \mathrm{kg}$ para óleos e gorduras virgens [5].

Resultados da avaliação sensorial do marinado de vongole armazenado constam na Tabela 4. Em relação à aparência do marisco (APAR), ficou evidenciado que as amostras apresentavam-se muito próximas do marisco ao natural. Quanto ao aroma de marisco (AROMAR) foi atribuído um valor médio, próximo de 5,0 (nem fraco/ nem forte). Os aromas ácido (AROACI) e avinagrado (AROVIN) foram de maior intensidade até aos 60 dias, com valores entre 4,0 e 5,0, mediana à fraca. O odor de ranço não foi detectado e, por isso, não indicado na Tabela 4.

Para o atributo sabor de marisco (SABMAR), foram atribuídas médias próximas de 5,0 (nem fraco/ nem intenso), enquanto em relação ao sabor ácido (SABACI), houve maior intensidade logo após o processamento, valor ao redor de 6,0 (ligeiramente intenso). Provavelmente isso seria devido à rápida pe- 
netração do ácido no produto, acentuada com o tratamento térmico. Aos 90 dias, ocorreu uma diminuição significativa do sabor ácido, atingindo valores próximos de 4,0, correspondendo à intensidade fraca deste atributo. O sabor rançoso não foi percebido pelos provadores. O sabor salgado (SABSAL) apresentou intensidade próxima ao valor médio (nem fraco/nem intenso), o mesmo ocorrendo com a textura (nem pouco/nem muito dura).

TABELA 4. Resultados médios $(n=15)$ da avaliação sensorial do marinado de vongole, durante 240 dias de armazenamento, a temperatura ambiente.

\begin{tabular}{|c|c|c|c|c|c|c|c|c|}
\hline \multirow{2}{*}{$\begin{array}{c}\text { Armazena- } \\
\text { mento } \\
\text { (dias) }\end{array}$} & \multicolumn{8}{|c|}{ Atributos } \\
\hline & APAR & AROMAR & AROACI & AROVIN & SABMAR & SABACI & SABSAL & DUREZA \\
\hline 01 & $9,0 \mathrm{~A}$ & $5,0 \mathrm{~A}$ & $4,0 \mathrm{~A}$ & $5,0 \mathrm{~A}$ & $5,0 \mathrm{~A}$ & $6,0 \mathrm{~A}$ & $6,0 \mathrm{~A}$ & $6,0 \mathrm{~A}$ \\
\hline 30 & $8,7 \mathrm{~A}$ & $4,6 \mathrm{~A}$ & $4,1 \mathrm{~A}$ & $4,9 \mathrm{~A}$ & $5,8 \mathrm{~A}$ & $5,5 \mathrm{~A}$ & $5,6 \mathrm{AB}$ & $5,4 \mathrm{~A}$ \\
\hline 60 & $8,6 \mathrm{~A}$ & $4,8 \mathrm{~A}$ & $4,2 \mathrm{~A}$ & $5,1 \mathrm{~A}$ & $5,2 \mathrm{~A}$ & $5,4 \mathrm{~A}$ & $5,5 \mathrm{AB}$ & $5,6 \mathrm{~A}$ \\
\hline 90 & $9,0 \mathrm{~A}$ & $4,7 \mathrm{~A}$ & $3,1 \mathrm{~B}$ & $4,8 \mathrm{~A}$ & $4,9 \mathrm{~A}$ & $4,3 \mathrm{~B}$ & $5,3 \mathrm{AB}$ & $5,2 \mathrm{~A}$ \\
\hline 120 & $9,0 \mathrm{~A}$ & $5,1 \mathrm{~A}$ & $3,1 \mathrm{~B}$ & $4,3 \mathrm{AB}$ & $5,5 \mathrm{~A}$ & $4,0 \mathrm{~B}$ & $5,1 \mathrm{BC}$ & $5,5 \mathrm{~A}$ \\
\hline 150 & $8,7 \mathrm{~A}$ & $4,5 \mathrm{~A}$ & $3,1 \mathrm{~B}$ & $4,3 \mathrm{AB}$ & $4,6 \mathrm{~A}$ & 4,1 B & $5,0 \mathrm{BC}$ & $5,3 \mathrm{~A}$ \\
\hline 180 & $8,9 \mathrm{~A}$ & $4,5 \mathrm{~A}$ & $3,1 \mathrm{~B}$ & $4,3 \mathrm{AB}$ & $5,0 \mathrm{~A}$ & 4,1 B & $5,1 \mathrm{BC}$ & $5,5 \mathrm{~A}$ \\
\hline 210 & $8,6 \mathrm{~A}$ & $4,6 \mathrm{~A}$ & $2,9 \mathrm{~B}$ & $4,4 \mathrm{AB}$ & $5,0 \mathrm{~A}$ & $4,2 \mathrm{~B}$ & $5,1 \mathrm{BC}$ & $5,5 \mathrm{~A}$ \\
\hline 240 & $8,5 \mathrm{~A}$ & $4,5 \mathrm{~A}$ & $2,9 \mathrm{~B}$ & $3,8 \mathrm{~B}$ & $4,7 \mathrm{~A}$ & $4,1 \mathrm{~B}$ & 4,4 C & $5,2 \mathrm{~A}$ \\
\hline
\end{tabular}

APAR: Aparência; AROMAR: Aroma Marisco; AROACI: Aroma Ácido; AROVIN: Aroma Vinagre; SABMAR: Sabor Marisco; SABACI: Sabor Ácido; SABSAL: Sabor Salgado; DUREZA: Textura.

Numa mesma coluna, médias com letras em comum não diferem significativamente entre si $(\mathrm{p}<0,05)$.

\section{4 - CONCLUSÕES}

O processamento proposto para a obtenção do marinado de vongole proporcionou ao produto desejável "esterilidade comercial", apreciável aceitabilidade e conveniente estabilidade durante 240 dias, quando armazenado a temperatura ambiente.

\section{5 - REFERÊNCIAS BIBLIOGRÁFICAS}

[1] AMERICAN PUBLIC HEALTH ASSOCIATION. Standart methods for the examination of dairy products. 13.ed., Washington D.C., 1984. 416 p.

[2] AOAC - ASSOCIATION OF OFFICIAL ANALYTICAL CHEMISTS. Official methods of analysis. 16 ed., Arlington, 1995. $1141 \mathrm{p}$.

[3] BARUFALDI, R.; OLIVEIRA, M.N. de. Fundamentos de Tecnologia de Alimentos. São Paulo: Livraria Atheneu, 1999. 317 p.

[4] BRASIL. Ministério da Saúde. Secretária Nacional de Vigilância Sanitária. Resolução $\mathrm{n}^{\circ} 12$, de 02 de janeiro de 2001. Dispõe sobre padrões microbiológicos. Diário Oficial [da] República Federativa do Brasil. Brasília, DF, 02 janl. 2001. Seção I, p. 48.

[5] BRASIL. Ministério da Saúde. Secretária Nacional de Vigilância Sanitária. Resolução n $\mathrm{n}^{\circ} 482$, de 03 de setembro de 1999. Dispõe sobre as características minimas de qualidade de óleos e gorduras vegetais. http:// www.anvisa.gov.br/legis/resol/482_01rdc.ntm

[6] DUTCOSKY, S.D. Análise sensorial de alimentos. Curitiba: Ed. DA Champagnat, 1996. 123.p.

[7] GAUlT, N.F.S. Marinaded Meat. Developments in Meat Science. v.5, p.191-246, 1991.

[8] GOMES, F.P. Curso de estatística experimental. 13.ed, São Paulo: Nobel, 1990. 468 p.

[9] INSTITUTO ADOLFO LUTZ. Normas Analiticas do Instituto Adolfo Lutz: métodos químicos e físicos para análise de alimentos. 3 ed., São Paulo, 1985. v. 1

[10] MEIlGAARD, M.; CIVILlE, G.V.; CARR, B.T. Sensory Evaluation Techiniques. Boca Raton Florida: $\mathrm{CbC}$ Press, v. 2, p. 40-44, 1987.

[11] MONTEIRO, C.L.B. Técnicas de avaliação sensorial. 2.ed. Curitiba: CEPPA-UFPR, 1984. 101p.

[12] PEDROSA, L.F.C.; COZZOLINO, S.M.F. Composição centesimal e de minerais de mariscos crus e cozidos da cidade de Natal/RN. Ciênc. Tecnol. Aliment., v. 21, n. 2, p. 154-157, 2001.

[13] STONE, H.; SIDEL, J.L. Sensory evaluation pratices. Florida, Academic Press, 1985. Cap.7: Affective testing, p. 227-252. 